\title{
ANALYSIS OF PLANE EXTRUDATE-SWELL OF HIGHLY ELASTIC LIQUIDS WITH MOLECULAR CONSTITUTIVE EQUATIONS
}

\author{
R.D. WESSON, T.C. PAPANASTASIOU * and J.O. WILKES \\ Department of Chemical Engineering, The University of Michigan, Ann Arbor, \\ Michigan 48109 (U.S.A.)
}

(Received March 4, 1988; in revised form January 3, 1989)

\section{Summary}

Plane, two-dimensional, polymer extrusion is analyzed by means of the Curtiss-Bird integral constitutive equation, streamlined finite-elements and Newton iteration. The Reynolds number is zero, the surface tension negligible and the melt does not slip at the wall. Starting from the Newtonian liquid of zero elasticity, the Newton iteration converged, within three to five iterations, up to a maximum Weissenberg number beyond 3500 . The predicted values of the die-swell at low elasticity are in agreement with those reported in the literature. At higher elasticities, the die-swell increases monotonically and levels off. Two other models examined, the Doi-Edwards and the Papanastasiou-Scriven-Macosko models, diverged at low Weissenberg numbers, however, the actual point of divergence was a function of the number of relaxation times. It appears that the second term of the Curtiss-Bird model, which incorporates the link tension coefficient, $\epsilon$, enhances significant numerical stability, in addition to the one due to the relaxation spectrum, as its different convergence behavior from the Doi-Edwards model implies.

\section{Introduction}

Numerical instability and divergence of the iterative method at high Weissenberg numbers is a common experience among investigators of com-

* To whom correspondence should be addressed. 
plex viscoelastic flow [1]. This was the primary focus of the "Workshop on Numerical Methods in non-Newtonian Fluid Mechanics" held at Arrowhead Lake, California (June, 1987). At this meeting, it became evident that a study of fundamentals was necessary in order to understand the difficulties encountered in complex viscoelastic flows.

The geometrical singularity in a contraction flow has been studied by Lipscomb et al. [2] who concluded that the flow is singular even at zero Weissenberg number and that the strength of the singularity increases with increasing Weissenberg number. Independently, Wesson and Papanastasiou [3] investigated the singularity in extrusion and concluded that slip alleviates the singularity but improves convergence only slightly (from $W e=1$ to $W e=2$ with the upper-convected Maxwell model). They attributed this to the fact that slip eliminates the stress singularity, but creates a maximum stress along a fluid particle's path, which was shown later to be equally catastrophic to the convergence of the Newton iteration.

To further validate the latter hypothesis, two simple flows with analytic solutions [4], a Poiseulle, channel-flow and a sink extensional flow, have been analyzed with several integral constitutive equations. The primary conclusions of this study were:

(a) Whenever the curve of the stress versus Weissenberg number of the analytic solution exhibited a maximum at a critical Weissenberg number, the Newton iteration of the finite-element equations diverged at exactly the same critical Weissenberg number.

(b) The most stable constitutive equation was the Curtiss-Bird model [5] in which the maximum stress was delayed to high Weissenberg numbers. The numerical solutions with this model existed at practically infinite Weissenberg number, increasing with $\epsilon$.

The generalization, then, to the two-dimensional, extrudate-swell flow was undertaken as described below. Modeling with integral constitutive equations with results and limitations similar to those with differential models began long ago [6-9]. An empirical constitutive equation designed by Papanastasiou et al. [10] was used recently by three groups with promising results. In [9] predictions have been obtained up to $W e=2$. Dupont and Crochet [11] predicted vortices in contraction flow at relatively low Weissenberg numbers, that had not been predicted by less realistic models. Very recently solutions have been obtained up to high Weissenberg numbers by the same model used in extrudate-swell flow by Luo and Tanner [12]. The Curtiss-Bird model has been used to analyze flows with corners at moderate Weissenberg numbers [8] and to study one-dimensional, fiber-spinning up to high Weissenberg numbers [13].

The advantages of the Curtiss-Bird model are:

(a) Molecular origin. 
(b) Parameters, calculated in principle from the molecular characteristics. Most importantly, no extensional data is needed for parameter estimation.

(c) A spectrum of relaxation times and a link-tension coefficient that extend the monotonicity of the stress with the strain-rate.

(d) Numerical stability in the one-dimensional flows examined: the channel shear-flow, the sink-flow and the fiber spinning flow.

Its disadvantages are a Newtonian-like behavior in the start-up of flow (stress jump) and the complicated kinematic tensors which are not in explicit relation to the velocity and the velocity gradient, which are the primary variables of flow. The former is not of a major concern in steady flows. The latter is resolved as explained in the next section.

\section{Governing equations}

The governing equations for creeping, incompressible flow are

$\nabla \cdot \boldsymbol{u}=0$

$\nabla(-p I+\tau)=0$

$\boldsymbol{n} \cdot \boldsymbol{u}=0, \quad \boldsymbol{n}=\frac{\nabla h}{|\nabla h|}$.

The third equation is the kinematic condition that forbids mass penetration across the line whose elevation from the midplane is $h$ and the unit normal is $n$. Thus, applied along a finite-element side, eqn. (3) makes the side a streamline segment. The streamlined finite-element method is detailed elsewhere [14]. The key idea in the streamlined finite element method is to solve eqn. (3) along the lateral sides of each element simultaneously with

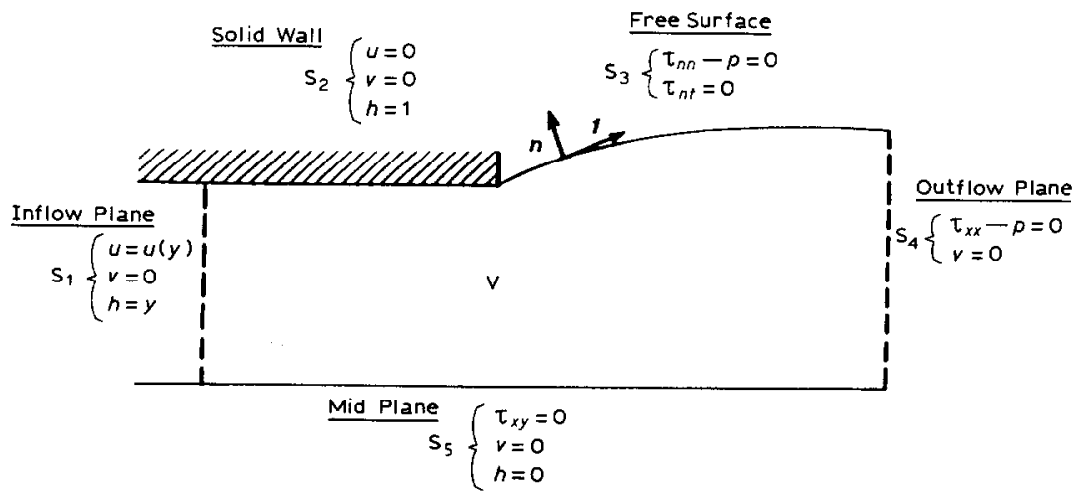

Fig. 1. Two-dimensional, plane extrudate-swell flow; geometry and boundary conditions. 
eqns. (1) and (2) (which are solved within the element). This forces the lateral sides to remain on streamline, which are in this way determined by their elevation $h$, in terms of the expansions by eqns. (4) to (6).

The viscous stress tensor, $\tau$, in eqn. (2) is given by the Curtiss-Bird equation, in terms of the velocity field, $\boldsymbol{u}$, as explained in the next section. The boundary conditions are shown in Fig. (1). The velocity profile, $u(y)$, at the inlet is calculated by a one-dimensional, finite-element analysis of the upstream, channel flow with the Curtiss-Bird model. This calculation is detailed elsewhere [4].

The unknowns are expanded in terms of finite-element basis functions: biquadratic for velocity; bi-linear for pressure; and quadratic for the streamline elevation from the midplane. The formal expansions are

$$
\begin{aligned}
& \boldsymbol{u}=\sum_{i=1}^{9} u_{i} \phi_{i}, \\
& p=\sum_{i=1}^{4} p_{i} \psi_{i}, \\
& h=\sum_{j=1}^{3} h_{j} \hat{\phi}_{j}(\eta=1) .
\end{aligned}
$$

Equations (1) to (3) are weighted integrally over the flow domain by the basis functions themselves. The surface integrals, resulting from the application of the divergence theorem, are either replaced by the boundary traction value or else the entire finite-element weighted, momentum equation is eliminated to impose a velocity or an elevation boundary condition. The resulting weighted residuals after the implementation of the boundary conditions are:

$$
\begin{aligned}
& R_{\mathrm{c}}^{i}=\int(\nabla \cdot u) \psi^{i} \mathrm{~d} V=0, \\
& \mathrm{R}_{\mathrm{M} x}^{i}=\int\left[\left(-p+\tau_{x x}\right) \frac{\partial \phi^{i}}{\partial x}+\tau_{x y} \frac{\partial \phi^{i}}{\partial y}\right] \mathrm{d} V=0, \\
& \mathrm{R}_{\mathrm{M} y}^{i}=\int\left[\left(-p+\tau_{y y}\right) \frac{\partial \phi^{i}}{\partial y}+\tau_{x y} \frac{\partial \phi^{i}}{\partial x}\right] \mathrm{d} V=0, \\
& R_{k}^{i}=\int \boldsymbol{n} \cdot \boldsymbol{u} \hat{\phi}_{i} \mathrm{~d} S=0 .
\end{aligned}
$$

The stresses $\tau_{i j}$, are determined explicitly by the integral constitutive equations in terms of the velocity values along the streamlines, defined by eqn. (10), as explained below. 


\section{Constitutive equation in terms of velocity}

The Curtiss-Bird equation has the form

$$
\begin{aligned}
\tau= & \int_{-\infty}^{t} m_{1}\left(t-t^{\prime}\right)\left(\phi_{1} C_{t}^{-1}\left(t^{\prime}\right)+\phi_{2} C_{t}\left(t^{\prime}\right)\right) \mathrm{d} t^{\prime} \\
& +\epsilon \int_{-\infty}^{t} m_{2}\left(t-t^{\prime}\right) G\left(C_{t}^{-1}\left(t^{\prime}\right), D(t)\right) \mathrm{d} t^{\prime},
\end{aligned}
$$

where the memory functions, $m_{1}$ and $m_{2}$, are defined as

$$
\begin{aligned}
& m_{1}\left(t-t^{\prime}\right)=\frac{96}{\lambda} \sum_{k=0}^{\infty} \exp \left[\frac{-(2 k+1)^{2}\left(t-t^{\prime}\right)}{\lambda}\right], \\
& m_{2}\left(t-t^{\prime}\right)=\frac{192}{\lambda^{2}} \sum_{k=0}^{\infty} \frac{1}{(2 k+1)^{2}} \exp \left[\frac{-(2 k+1)^{2}\left(t-t^{\prime}\right)}{\lambda}\right] .
\end{aligned}
$$

In this form the equation is not usable because the relations of $\phi_{1}, \phi_{2}$ and $\boldsymbol{G}$ to the kinematics are given through complicated, elliptic integrals. Fortunately Currie [15] linearized these functions in terms of the Finger and Cauchy tensors, $C_{t}^{-1}\left(t^{\prime}\right)$ and $C_{t}\left(t^{\prime}\right)$, and their invariants, $I$ and $I I$, such that

$\dot{\phi}_{1}=\frac{5}{J-1}$,

$\phi_{2}=\frac{-5(I I+3.25)^{-1 / 2}}{J-1}$,

where

$$
J=I+2(I I+3.25)^{1 / 2} \text {. }
$$

The components of the function $\boldsymbol{G}$ were approximated by Currie [15] by

$$
G_{i j}\left(C_{t}^{-1}\left(t^{\prime}\right), D(t)\right)=C_{i j k m} D_{k m}(t),
$$

where

$$
\begin{aligned}
C_{i j k m}= & -U_{11} C_{i j}^{-1} C_{k m}^{-1}-U_{12}\left(I I C_{i j}^{-1} \delta_{k m}-C_{i j}^{-1} C_{k m}-C_{k m}^{-1} C_{i j}\right) \\
& +U_{22} C_{i j}\left(I I \delta_{k m}-C_{k m}\right)-V_{1} C_{k m}^{-1} \delta_{i j}-V_{2}\left(I I \delta_{k m}-C_{k m}\right) \delta_{i j} \\
& +\frac{1}{2} V\left(\delta_{i j} \delta_{j m}+\delta_{j k} \delta_{i m}\right)-\frac{1}{2} U_{2}\left(\delta_{j m} C_{i k}+\delta_{j k} C_{i m}+\delta_{i k} C_{j m}+\delta_{i m} C_{j k}\right) \\
& +B_{i j} \delta_{k m},
\end{aligned}
$$

and

$$
B_{i j}=\left(I I U_{12}-V_{1}\right) C_{i j}^{-1}+\left(V_{2}-I I U_{22}\right) C_{i j}+\frac{1}{3}\left(I V_{1}+2 I I V_{2}\right) \delta_{i j},
$$


with

$U=\left(5 \ln \left[I+2[I I+3.25]^{1 / 2}-1\right]\right) / 7$,

$V=\left(5-I U_{1}+I I U_{2}\right) / 3$,

and

$U_{11}=\partial^{2} U / \partial I^{2}, \quad V_{1}=\partial V / \partial I$, etc.

The Finger, the Cauchy and the current or local rate-of-strain, $D(t)$, tensors can be expressed in terms of past and present velocities along streamlines by utilizing a moving system of coordinates conforming to the streamlines [16], or by solving the deformation gradient equation analytically in a protean [17] coordinate system [18] and then transforming the results to the cartesian system. The resulting expressions are from [18]:

$$
\begin{aligned}
& \left(C^{-1}\right)_{11}=\left(\frac{u}{Q}\right)^{2}+\left(\frac{Q}{q}\right)^{2}\left(q u \Omega-\frac{v}{q}\right)^{2}, \\
& \left(C^{-1}\right)_{12}=\frac{u v}{Q^{2}}+\left(\frac{Q}{q}\right)^{2}\left(q u \Omega-\frac{v}{q}\right)\left(q v \Omega+\frac{u}{q}\right),
\end{aligned}
$$

and

$$
\left(C^{-1}\right)_{22}=\left(\frac{v}{Q}\right)^{2}\left(\frac{Q}{q}\right)^{2}\left(q v \Omega+\frac{u}{q}\right)^{2}
$$

where

$$
\begin{aligned}
& \Omega=\int_{t^{\prime}}^{t} \frac{2}{q^{4}}\left[u v\left(D_{22}-D_{11}\right)+\left(u^{2}-v^{2}\right) D_{12}\right] \mathrm{d} t^{\prime}, \\
& Q=\sqrt{U^{2}+V^{2}}, \\
& q=\sqrt{u^{2}+v^{2}}, \\
& \text { and }
\end{aligned}
$$

$$
C_{t}^{-1}\left(t^{\prime}\right) C_{t}\left(t^{\prime}\right)=I \text {. }
$$

The integral represented by $\Omega$ is a measure of the accumulated strain between past velocities, $U\left(t^{\prime}\right)$ and $V\left(t^{\prime}\right)$, and present velocities, $u(t)$ and $v(t)$, and is evaluated along streamlines. This conforms nicely to the streamlined finite element method which was developed for this purpose [14]. 


\section{Finite-element analysis}

The streamlined elements are mapped by the isoparametric mapping

$x=\sum_{i=1}^{9} x_{i} \phi_{i}(\xi, \eta)$

$y=\sum_{i=1}^{9} y_{i} \phi_{i}(\xi, \eta)$

$h=\sum_{i=1}^{3} h_{i} \phi_{i}(\xi, \eta=1)$,

into the basic square $Q(\xi, \eta)$, where $-1<\eta<1$ and $-1<\xi<1$. The derivatives and integrals of the weighted residuals in the actual flow domain, of coordinates $x$ and $y$, are transformed and evaluated on the basic square, of coordinates $\xi$ and $\eta$, by means of the Jacobian of the transformation

$\left[\begin{array}{c}\frac{\partial(\quad)}{\partial x} \\ \frac{\partial(\quad)}{\partial y}\end{array}\right]=\left[\begin{array}{ll}\frac{\partial \xi}{\partial x} & \frac{\partial \eta}{\partial x} \\ \frac{\partial \xi}{\partial y} & \frac{\partial \eta}{\partial y}\end{array}\right]\left[\begin{array}{l}\frac{\partial()}{\partial \xi} \\ \frac{\partial()}{\partial \eta}\end{array}\right]$.

The volume integral, $\mathrm{d} V$, is transformed as

$\mathrm{d} V=\mathrm{d} x \mathrm{~d} y=|M| \mathrm{d} \xi \mathrm{d} \eta$,

where $|M|$ is the determinant of the Jacobian, given by

$M=\left[\begin{array}{ll}\frac{\partial x}{\partial \xi} & \frac{\partial y}{\partial \xi} \\ \frac{\partial x}{\partial \eta} & \frac{\partial y}{\partial \eta}\end{array}\right]$.

Similarly, the surface integral, $\mathrm{d} S$, along a streamline, which is made-up by segments of finite-element lateral sides, becomes

$\mathrm{d} S=\sqrt{\left(\frac{\partial x}{\partial \xi}\right)^{2}+\left(\frac{\partial y}{\partial \xi}\right)^{2}} \mathrm{~d} \xi$,

along the straight line $\eta=0$ of the basic square $Q(\xi, \eta)$. The calculations involved in eqns. (33) to (36) are trivial given the isoparametric mapping defined by eqns. (30) to (32).

The domain is discretized into elements as shown in Fig. 2. This tessellation gives an apparent mesh-independent solution, according to Fig. 4, down to $0.1 \times 0.2$ element at the singularity where the method diverged, as it 

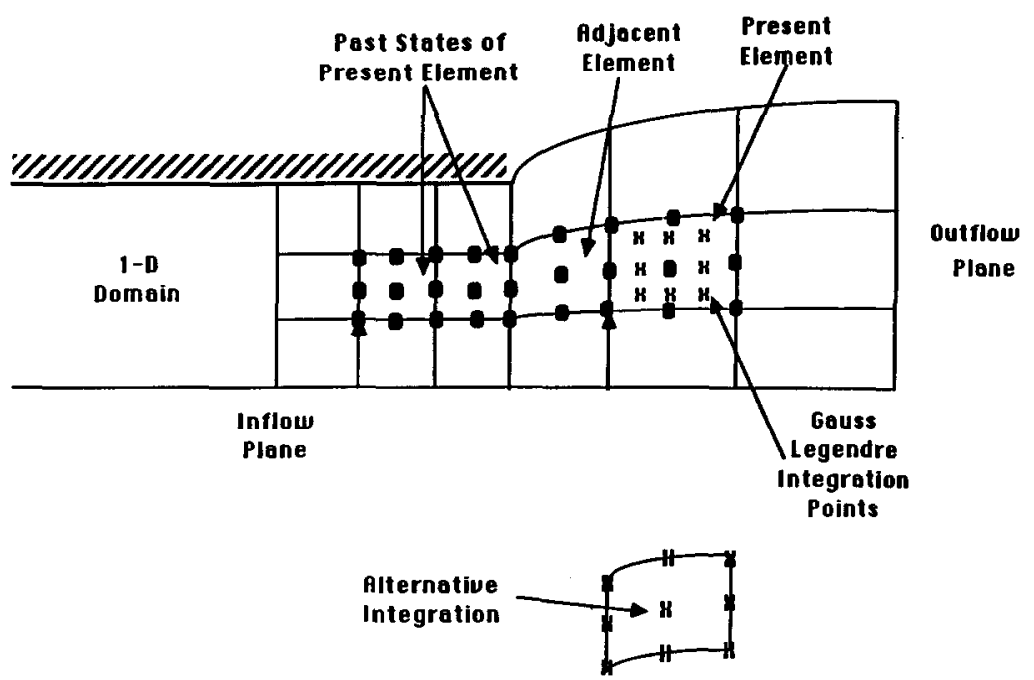

Fig. 2. Present, adjacent and upstream elements for stress calculation.

should [3]. Because of the streamlined tessellation, the mesh around the singularity becomes finer and finer, down to $0.1 \times 0.2$, as the Weissenberg number increases. This tendency is obvious in Fig. 3. The stress in each element is calculated from far upstream, sometimes from outside the flow domain depending on the memory of the fluid, along streamlines made-up by element lateral sides. The calculations are facilitated further by the fact that the streamlines become straight lines on the isoparametric domain $(\xi$, $\eta)$.

The exponential travelling times

$t-t^{\prime}=\int_{x^{\prime}}^{x} \frac{\mathrm{d} \alpha}{u(\alpha)}$

and the accumulated deformation, (eqn. (26)), are additive; therefore, they are evaluated and stored immediately after each Newton iteration in an appropriate matrix and are retracted appropriately for the evaluation of the residuals of the next Newton iteration.

A nine-point Gauss-Legendre quadrature was used to evaluate the stresses in each element. The stress in an element was obtained by summing-up the individual contributions from the upstream elements. The stress contribution from elements far upstream, with respect to the element being assembled, is small compared to the contributions from its neighboring elements. Therefore, the nine-point Gauss-Legendre scheme successfully approximated the stress contributions from elements far upstream. The current and its adjacent upstream element in the same streamtube were first subdivided 
into segments, depending on the magnitude of the relaxation time, and then the nine-point quadrature was applied to each segment to obtain a more accurate stress approximation.

An alternative integration scheme, less accurate for the current element, but more accurate for the evaluation of the memory integrals along streamlines is shown in Fig. 2. It is also cost efficient because the required values of the velocity and the streamline elevation at the integration points coincide with the nodal values, which are directly available and thus no interpolation by means of the nine basis functions is required. Results with this scheme are currently being evaluated.

The streamlined elements facilitate, actually eliminate, particle tracking. The particle is forced to be on a streamline whose position, coinciding with element lateral sides, is known from the previous Newton iteration. In addition, the streamlined finite-element method facilitates bookkeeping and makes Newton iteration possible, which brings with it the Jacobian for continuation and linear stability analysis [19].

In the Newton iteration

$\boldsymbol{J}\left(\boldsymbol{q}^{N}\right)\left[\boldsymbol{q}^{N+1}-\boldsymbol{q}^{N}\right]=-\boldsymbol{R}\left({ }^{N}\right)$,

$q=\left(u_{1} v_{1}, p_{1}, h_{1}, \ldots, u_{\mathrm{n}}, v_{\mathrm{n}}, p_{\mathrm{k}}, h_{\mathrm{m}}\right]$ is the vector of all the unknowns including the streamline elevation, $h_{i}, \boldsymbol{R}$ is the residual vector and $\boldsymbol{J}$ the Jacobian. The entries of the Jacobian matrix are evaluated analytically whenever possible. If the complexity of the residual prohibits a cost effective, analytic determination of the derivatives, then the Jacobian entry is evaluated numerically. The numerical approximations were computed by

$J_{i j}=\frac{\partial R^{i}}{\partial q^{j}}=\left[\frac{r^{i}\left(q^{j}+\Delta q^{j}\right)-R^{i}\left(q^{i}\right)}{\Delta q^{j}}\right]_{q_{k \neq i}}$,

where $\Delta$ represents an infinitesimal variation defined here as

$\Delta q^{j}=10^{-4} q^{j}$.

The derivatives of the viscoelastic stress in the momentum equations with respect to the nodal positions and velocities were computed in this way. The rest derivatives, of the momentum with respect to pressure, of the continuity with respect to the current velocities and the current location and of the kinematic equation with respect to the current streamline velocities and position, were computed analytically.

Existing frontal subroutines [20] can not be utilized to solve the lower triangular structure of the Jacobian which arises from the sensitivity of the current or local stress with respect to the upstream nodal velocities and displacements. However, this is not a serious drawback because the cost to 
solve the linear system is very small compared to the cost of filling the Jacobian matrix (approximately $1: 10$ ). Roughly seven minutes per Newton iteration were required for convergence at the highest Weissenberg numbers on the San Diego Cray X-MP/48 supercomputer. Once the backward memory of the elements adjacent to the outlet reached the inlet line, no significant cost increase was observed. The total cost per run was reduced from approximately 30 to 20 minutes by implementing the most elementary quasi-Newton iteration.

\section{Results}

The Weissenberg number here is defined by

$W e=\dot{\gamma}_{w} \lambda$,

where $\dot{\gamma}_{\mathrm{w}}$ is the shear rate at the solid wall away from the exit, and $\lambda$ is the maximum relaxation time. Other definitions of $\lambda$ are possible such as (based on five relaxation time used):

(a) average relaxation time for $\epsilon=0$, defined as

$\bar{\lambda}=\frac{\sum_{1}^{5} a_{k} \lambda_{k}^{2}}{\sum_{1}^{5} a_{k} \lambda_{k}}=0.9 \lambda$,

where $a_{k}=96 / \lambda$ in eqn. (12).

(b) average relaxation time for $\epsilon \neq 0$, defined as

$\lambda_{\epsilon}=\frac{\bar{\lambda}+\epsilon \bar{\lambda}_{\epsilon}}{1+\epsilon}=\lambda\left(\frac{0.9+\epsilon}{1+\epsilon}\right)$,

where $\bar{\lambda}_{\epsilon}$ is the average relaxation time of the second integral term or eqn. (13). The Weissenberg number of the reported results was calculated by means of eqn. (42) and is close to those calculated by means of eqns. (41) and (43).

For the results reported, the Newton iteration converged quadratically within three to five iterations, to within a maximum error of $10^{-4}$ in the unknowns, between consecutive iterations. Zero order continuation was used, i.e. the converged solution at a Weissenberg number was taken as the initial estimation to the Newton iteration at the higher Weissenberg number. The tessellation shown in Fig. 3 produced the value of 1.20 , widely reported in the literature for the extrudate-swell of the Newtonian liquid and tessellation independent solutions, according to the error criterion used, for $W e \neq 0$. The tessellation does not have to extend further upstream because the 

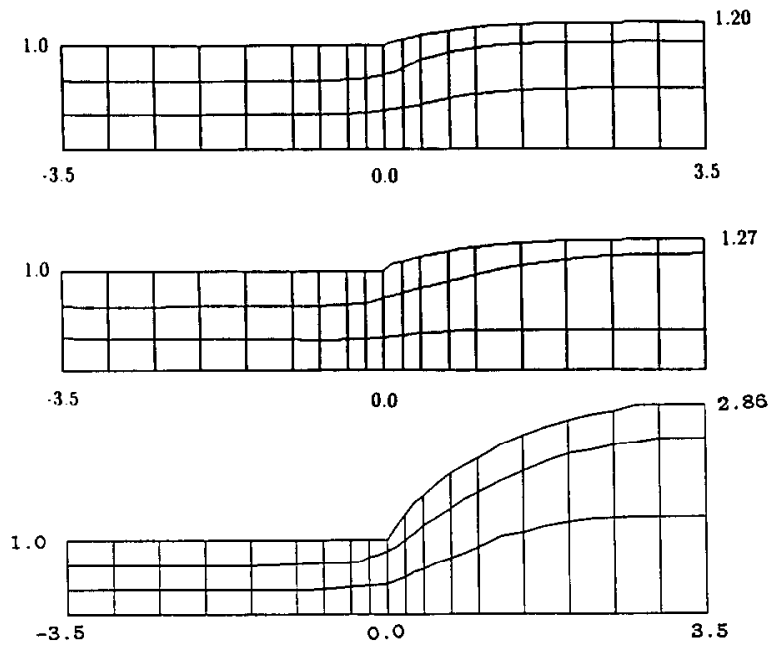

Fig. 3. Finite-element tessellations after convergence. (a) Newtonian liquid, (b) Maxwell liquid at $W e=0.96$, (c) Curtiss-Bird liquid at $W e=3470$ (with $\epsilon=0.5$ ).

excessive memory is accommodated by the one-dimensional, channel flow approximation upstream from the inlet.

The Lodge model [21], which is equivalent to the upper-convected Maxwell model, produced results identical to those obtained by other investigators [7,22-24] as shown in Fig. 4.

The Papanastasiou-Scriven-Macosko model, with one relaxation time, produced results up to a Weissenberg number of 0.98 . With three relaxation times the critical Weissenberg number was 3.17 (Fig. 5). Thus, the conclu-

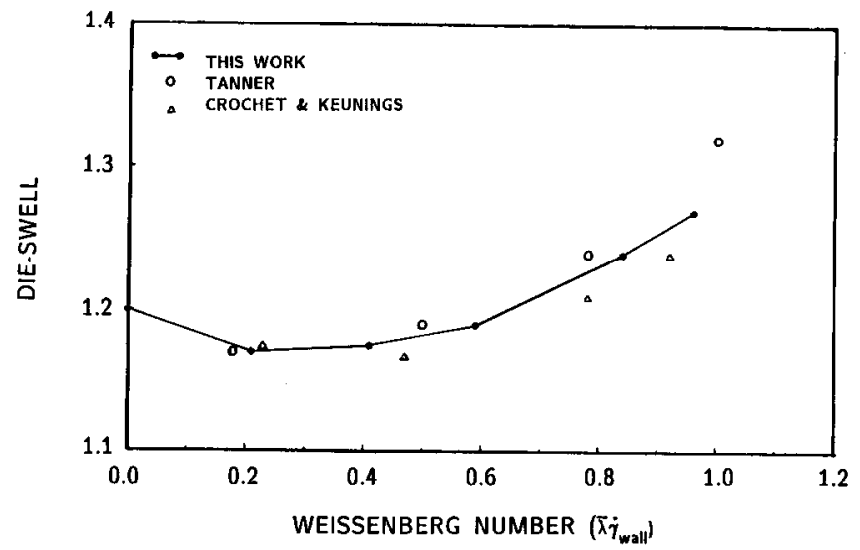

Fig. 4. Die-swell predictions with the upper-convected Maxwell model and comparison of the predictions to those of other investigators [22,23]. 


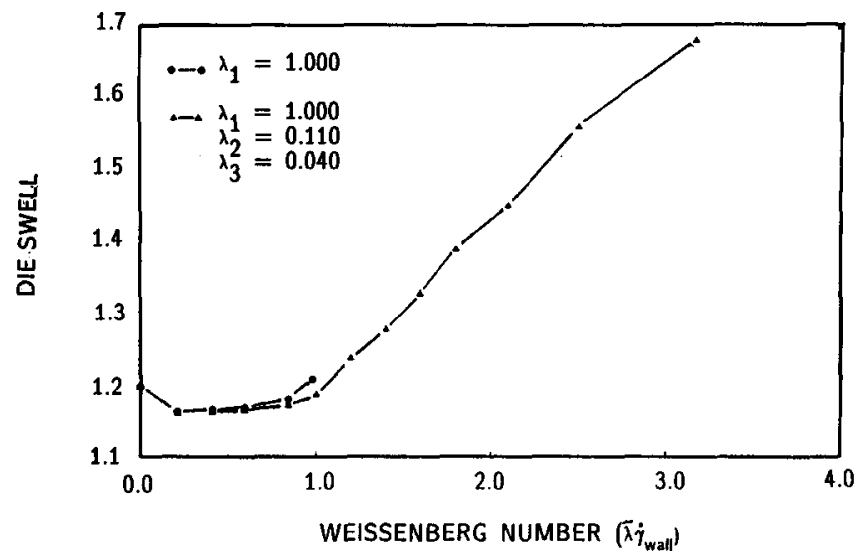

Fig. 5. Die-swell predictions with the Papanastasiou-Scriven-Macosko model $[9,10]$ with one and with three relaxation times.

sion from these results is that a spectrum of relaxation times enhances numerical stability which results in solutions at higher Weissenberg numbers. The recent achievement of solutions at high elasticity by Luo and Tanner [12] with the same model and eight relaxation times appears to be in agreement with these findings. The Doi-Edwards model [25] was almost identical to the Papanastasiou-Scriven-Macosko model as shown by Fig. 6 .

Figure 7 illustrates the predicted die-swell of a Curtiss-Bird fluid at several Weissenberg numbers. With $\epsilon=0$, the Curtiss-Bird model reduces to the Doi-Edwards model and the Newton iteration diverged at $W e=3.46$. For $\epsilon \neq 0$ the Newton iteration converged up to $W e=3470$, where we

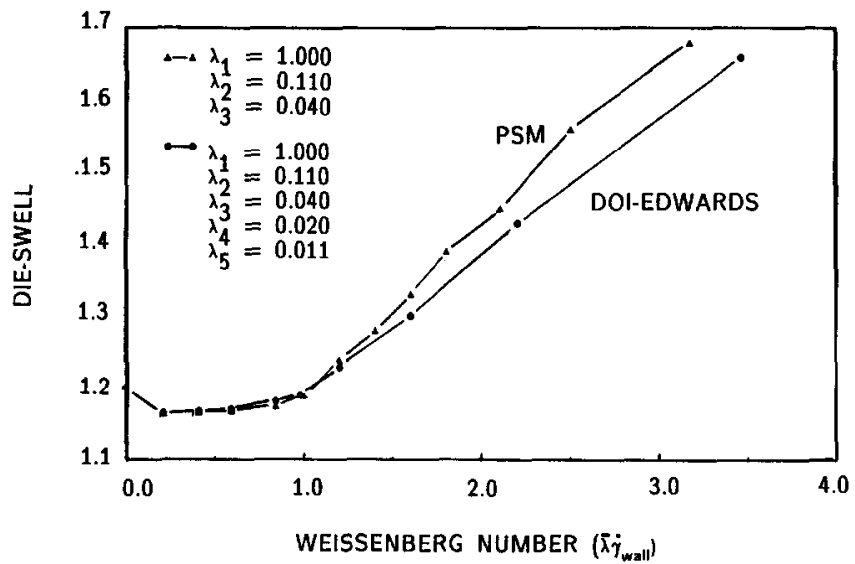

Fig. 6. Comparison of the die-swell predictions of the Doi-Edwards and the PapanastasiouScriven-Macosko models. 


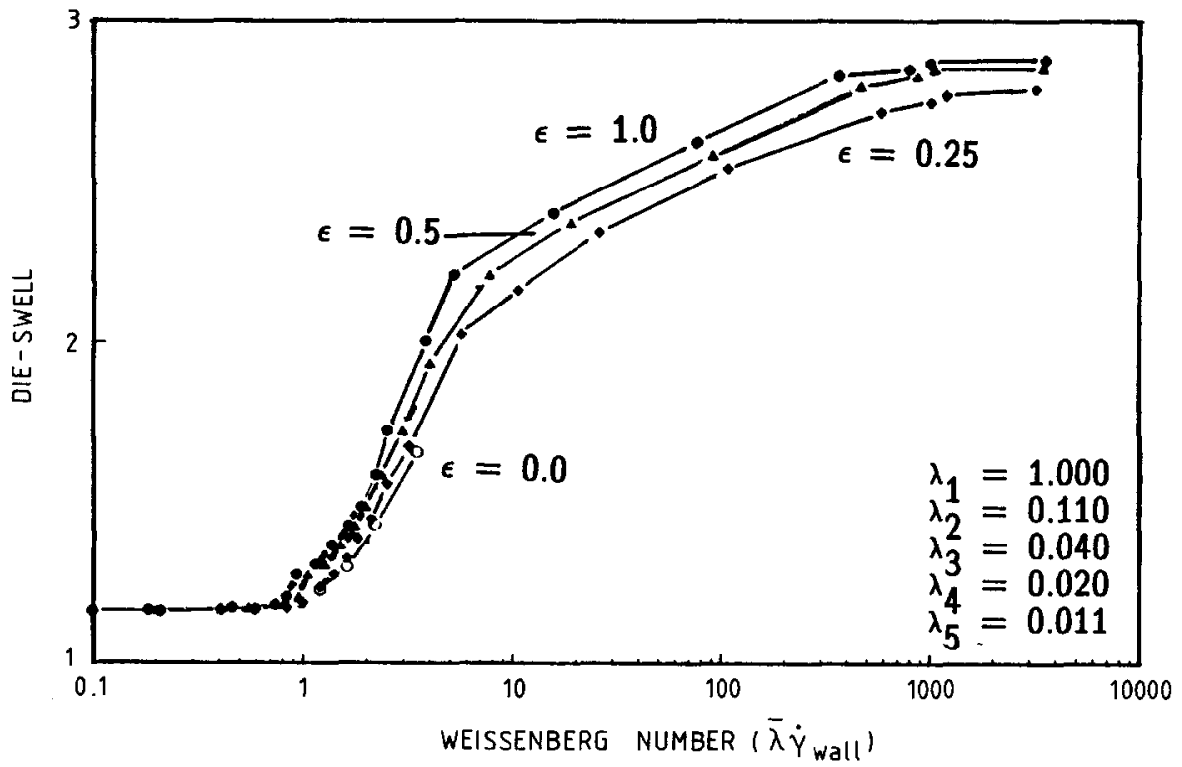

Fig. 7. Die-swell predictions with the Curtiss-Bird model with increasing Weissenberg numbers and with several link-tension coefficients.

discontinued the calculations. The predictions at $W e<1$ agreed with those of the upper-convected Maxwell model as shown by Fig. 4.

The predicted die-swell begins to level off at approximately 500. It is not known wether this trend is real since no experimental data for sheet extrusion are available at this elasticity. The curve up to $W e=1000$ was obtained with $\lambda=1 \mathrm{~s}$ at several channel pressure drops. The curve was continued further by increasing the relaxation time at constant pressure drop.

Figure 8(a) shows the centerline velocity distributions along the flow direction at several Weissenberg numbers. An isolated view of the centerline velocity at the maximum Weissenberg number of 3470 is shown in Fig. 8(b). The velocity inside the channel remains relatively constant and decreases upon exiting the channel. The corresponding pressure distributions for the predicted velocities are shown in Fig. 9. The used scales mask some tessellation-dependent wiggles in the velocity and the pressure in the viscinity of the singularity at the exit of the channel of amplitude up to $5 \%$ of the smoothened values. These wiggles are artificial, stemming from the exit singularity, as demonstrated by several investigators.

A velocity profile inside the channel is shown in Fig. 10 for a Newtonian liquid and a Curtiss-Bird liquid at $W e=3470$ at dimensionless pressure drop $P D / \mu V=2500$ and $L / D=3.5$. The Curtiss-Bird fluid exhibits the 

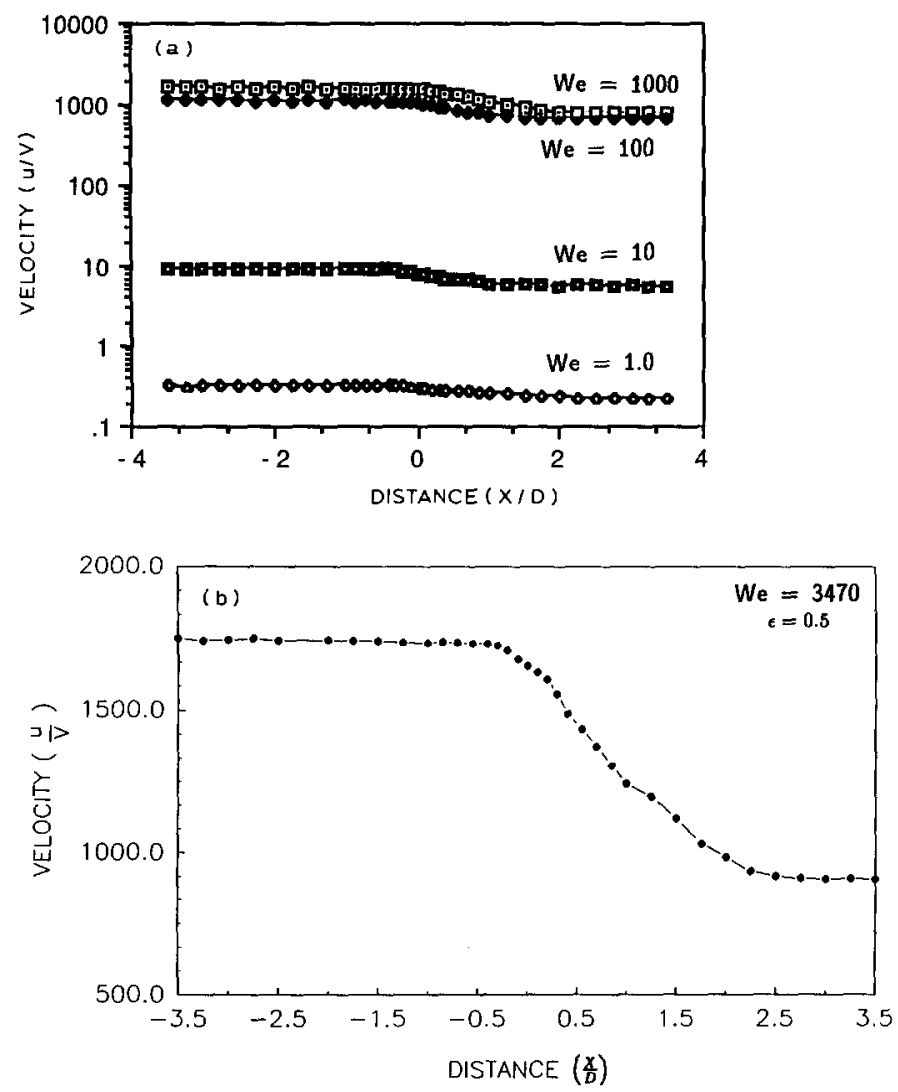

Fig. 8. Centerline velocity distributions. (a) At several Weissenberg numbers, (b) at Weissenberg number of 3470 .

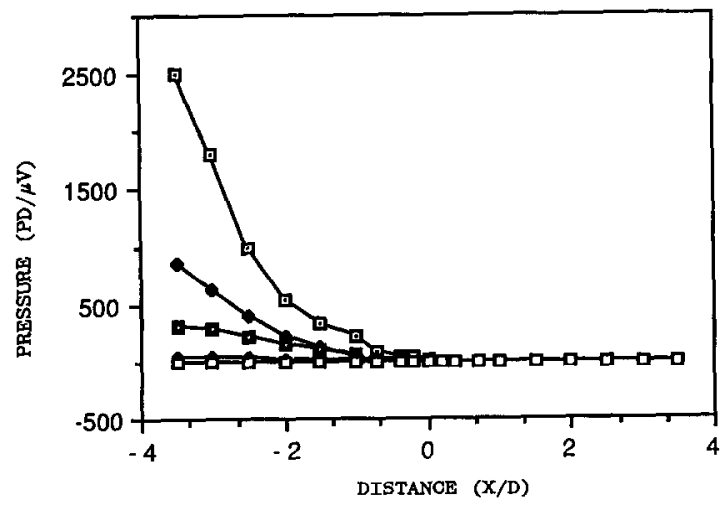

Fig. 9. Pressure distributions corresponding to Weissenberg numbers and centerline velocity values of Fig. 8. $\square W e=3470, \diamond W e=1000, \square W e=100, \diamond W e=10, \square W e=1.0$, and $\square$ $W e=0.1$. 


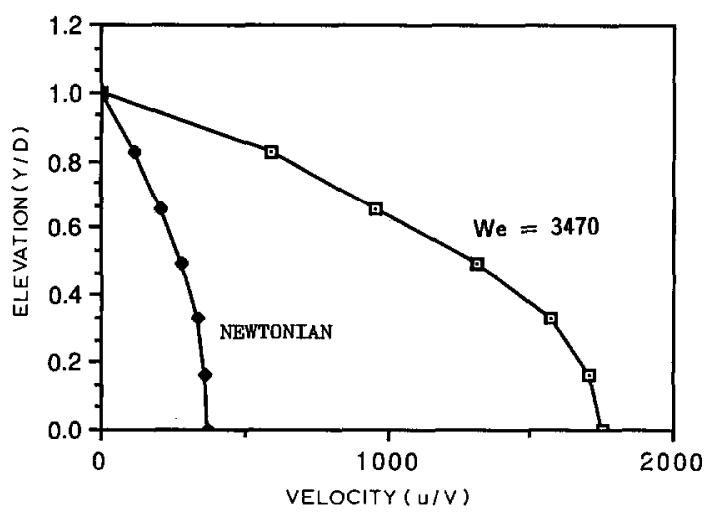

Fig. 10. Channel-flow, velocity profiles for a Curtiss-Bird fluid of $\epsilon=0.5$ and for Newtonian liquid at common dimensionless pressure drop, $P D / \mu V=2500$, and channel aspect ratio, $L / D=3.5$.

characteristic shear-thinning behavior and therefore increased velocity at the same pressure drop.

In two previous papers $[3,4]$ we concluded that avoiding the stress maximum by a constitutive equation with a spectrum of relaxation times and by some mechanism of slip could be helpful in viscoelastic calculations. Analysis of simple flows showed that the Curtiss-Bird model may fulfill these demands, being stable up to high elasticities. The extrudate-swell analysis appears to confirm these hypotheses.

It is now evident that convergence can be improved by using a spectrum of relaxation times, not just one. This may be true of differential models too, since most modeling with these equations had included a single relaxation time. This will ultimately be essential in order to predict realistic results for liquids exhibiting many relaxation times [26]. In addition it appears that the link-tension coefficient, $\epsilon$, initiates an unknown mechanism, not just a Newtonian viscosity, which stabilized the solution even at values of $\epsilon$ close to but not equal to zero; a Newtonian viscosity added to an integral equation or incorporated into a differential model as a retardation constant improves convergence only slightly [3].

Comparisons of the predictions of the Curtiss-Bird model with data on monodisperse polymers in plane extrusion is needed. The model predictions in shear and elongation are quite good [13,27-29]. The present work provides a good opportunity to validate the model in mixed deformations as well.

\section{Conclusions}

Viscoelastic computations at high Weissenberg number have been achieved by a scheme based on: 
(i) the Curtiss-Bird integral constitutive equation for monodisperse melts and concentrated solutions,

(ii) streamlined finite-elements that eliminate particle tracking, and

(iii) Newton iteration.

The predicted die-swell at low elasticity agrees with the findings of other investigators. Starting from the Newtonian value of 1.20 , the die-swell goes through a minimum at $W e=0.3$, then increases monotonically and levels off beyond $W e=500$. Data at high elasticity are required to further validate the predictions. Numerical stability was enhanced by the relaxation spectrum and by the link tension coefficient of the Curtiss-Bird model which appears to induced an unknown stabilizing mechanism.

The required computing time per run needs to be improved. Current optimization attempts, in addition to vectorization, include a numerical integration scheme with points on streamlines, Broyden's iteration and analytical evaluation of more Jacobian entries to replace the high-cost, numerical evaluation. The work is currently being extended to axisymmetric extrusion and to extrusion under tension as in fiber-spinning.

\section{Acknowledgements}

This research was supported by a grant from the Reaction and Process Engineering Program of the National Science Foundation-Grant CBT 8504607, and by the San Diego Supercomputer Center.

\section{References}

1 M.J. Crochet and K. Walters, Ann. Rev. Fluid Mech., 15 (1983) 241.

2 G.G. Lipscomb, R. Keunings and M.M. Denn, J. Non-Newtonian Fluid Mech., 24 (1987) 85.

3 R.D. Wesson and T.C. Papanastasiou, J. Non-Newtonian Fluid Mech., 26 (1988) 277.

4 R.D. Wesson, T.C. Papanastasiou, and J.O. Wilkes, J. Rheol., in press.

5 C.F. Curtiss and R.B. Bird, J. Chem., Phys., 74 (1981) 2026.

6 H. Court, A.R. Davies and K. Walters, J. Non-Newtonian Fluid Mech., 8 (1981) 95.

7 B. Caswell and K. Viriyayuthakorn, J. Non-Newtonian Fluid Mech., 12 (1983) 12.

8 B. Bernstein, D.S. Malkus and E.T. Olsen, Int. J. Numer. Meth. Fluid, 5 (1985) 43.

9 T.C. Papanastasiou, L.E. Scriven and C.W. Macosko, J. Non-Newtonian Fluid Mech., 22 (1987) 271.

10 T.C. Papanastasiou, L.E. Scriven and C.W. Macosko, J. Rheol., 27 (1983) 387.

11 S. Dupont and M.J. Crochet, J. Non-Newtonian Fluid Mech., 29 (1988) 81.

12 X.-L. Luo and R.I. Tanner, J. Non-Newtonian Fluid Mech., 22 (1986) 61.

13 Z. Chen and T.C. Papanastasiou, Int. Pol. Proc., 2 (1987).

14 T.C. Papanastasiou, C.W. Macosko and L.E. Scriven, in: R.H. Gallagher, G.F. Garey, J.T. Olsen and O.C. Zienkiewicz (Eds.), Finite Elements in Fluids, Vol. 6. John Wiley and Sons, New York, NY, 1985, p. 262.

15 P.K. Currie, J. Non-Newtonian Fluid Mech., 11 (1982) 53. 
16 H.H. Winter, J. Non-Newtonian Fluid Mech., 10 (1982) 157.

17 J.L. Duda and J.S. Vrentas, Chem. Eng. Sci., 22 (1966) 855.

18 K. Adachi, Rheol. Acta, 22 (1983) 326.

19 R.A. Brown, L.E. Scriven and W.J. Silliman, in: P.J. Holmes (Ed.), New Approaches to Non-Linear Problems in Dynamics. Soc. Ind. Appl. Math., Philadelphia, PA, 1980, p. 298.

20 P. Hood, Int. J. Numer. Meth. Eng., 10 (1986) 120.

21 A.S. Lodge, Trans. Faraday Soc., 52 (1956) 120.

22 M.J. Crochet and R. Keunings, J. Non-Newtonian Fluid Mech., 10 (1982) 339.

23 X.-L. Luo and R.I. Tanner, J. Non-Newtonian Fluid Mech., 22 (1986) 179.

24 P.W. Chang, T.W. Patten and B.A. Finlayson, Comput. Fluids, 6 (1979) 284.

25 N. Phan-Thien, J. Rheol., 22 (1978) 259.

26 M. Doi and S.F. Edwards, J. Chem. Sci. Faraday Trans., 74 (1979) 918.

27 R.B. Bird, H.H. Saab and C.F. Curtiss, J. Chem. Phys., 76 (1982) 1102.

28 R.B. Bird, H.H. Saab and C.F. Curtiss, J. Chem. Phys., 77 (1982) 4747.

29 H.H. Saab, R.B. Bird and C.F. Curtiss, J. Chem. Phys., 77 (1982) 4758. 